\title{
How can better identification of T790M help to inform treatment sequencing decisions in EGFR mutation-positive non-small-cell lung cancer?
}

\author{
Maximilian J Hochmair*,1 \\ ${ }^{1}$ Department of Respiratory \& Critical Care Medicine \& Ludwig Boltzmann Institute for COPD \& Respiratory Epidemiology, Otto \\ Wagner Hospital, 1140 Vienna, Austria \\ *Author for correspondence: Tel.: +43 191060 41240; Fax: +43 191060 41249; maximilian.hochmair@wienkav.at

\section{"Optimizing the detection of T790M after progression on first-/second-generation TKIs should allow more patients to benefit from subsequent osimertinib treatment."}

First draft submitted: 14 March 2019; Accepted for publication: 27 March 2019; Published online:

29 April 2019

Keywords: EGFR • sequencing • T790M

The availability of three generations of EGFR tyrosine kinase inhibitors (TKIs) with different clinical profiles has revolutionized the treatment of $E G F R$ mutation-positive $(E G F R \mathrm{~m}+)$ non-small-cell lung cancer (NSCLC). Compared with the first-generation EGFR TKIs, erlotinib and gefitinib, head-to-head trials have shown improved efficacy with the second-generation irreversible ErbB family blockers, afatinib and dacomitinib, and the thirdgeneration irreversible EGFR wild-type sparing TKI, osimertinib [1-3]. Unfortunately, despite high initial response rates with EGFR TKIs, acquired resistance is inevitable regardless of the choice of first-line TKI. Although other mechanisms exist, the discovery that the predominant mechanism of resistance to first-and second-generation EGFR TKIs is the 'gatekeeper' T790M mutation ( 50-70\% of cases) [4,5] prompted the development of third-generation, T790M-directed EGFR TKIs. Osimertinib, which is active against both EGFR-sensitizing and T790M-resistance mutations, was initially approved in the second-line setting based on remarkable activity in patients with metastatic $E G F R \mathrm{~m}+\mathrm{NSCLC}$ after failure of first-line TKIs [4,6].

Given that osimertinib use in second line requires the presence of T790M, it is imperative for tumors to be characterized for molecular aberrations at the first manifestation of progression, to inform decisions about appropriate subsequent therapy [7]. Optimizing the detection of T790M after progression on first-/second-generation TKIs should allow more patients to benefit from subsequent osimertinib treatment [8]. Liquid biopsies offer the potential for easier, noninvasive mutation testing compared with tissue biopsies, which can also be challenging in clinical practice due to patient ineligibility, tumor inaccessibility and insufficient tissue availability [8]. Additionally, liquid biopsy avoids challenges associated with tumor heterogeneity, which can mean that mutations are missed if tissue biopsy is taken from a single tumor region [9]. On the other hand, tissue biopsy offers histological analysis of the tumor, and is generally more sensitive than current liquid biopsy assays which can produce false-negative results. Consequently, current guidelines recommend that negative findings by liquid biopsy should be followed-up by confirmatory tissue rebiopsy [10]. Where possible, all possible detection methods should be employed, using the most sensitive technique(s) available. In this regard, methods such as droplet digital PCR (ddPCR) and next-generation sequencing offer greater sensitivity and specificity compared with liquid biopsy methods [8,11].

In a recent analysis using both liquid biopsy (ddPCR) and tissue rebiopsy, the T790M mutation was identified in 49/67 patients (73\%) who progressed on afatinib, including 36/54 patients (67\%) after first-line afatinib [8]. This is consistent with previous reports which estimated the prevalence of T790M to be approximately 50-70\% following progression on first-generation EGFR TKIs or afatinib [4,5]. The ddPCR technique used in the recent study was highly sensitive, which is likely to explain the high rate of T790M detection [8]. Indeed, a separate analysis comparing ddPCR with the US FDA-approved, semiquantitative PCR-based Cobas ${ }^{\circledR}$ EGFR Mutation Test v2

Future $\because$ Medicine 
found generally high concordance, but the T790M positivity rate in patients who progressed during EGFR TKI treatment was $66 \%$ by ddPCR compared with only $24 \%$ according to the Cobas test [12]. Ultimately, these findings support the hypothesis that most patients will develop T790M-acquired resistance following progression on afatinib and will be eligible for subsequent osimertinib treatment, providing the right test is used. Subsequent osimertinib treatment in patients with T790M-acquired resistance to first-/second-generation EGFR TKIs achieved response rates of $>70 \%$, independent of T790M copy number $[7,8]$. These are important findings as they suggest that second-line osimertinib may be effective even in patients with subclonal T790M-positive tumors [8].

Despite the lack of prospective data on sequential EGFR-TKI treatment, retrospective analyses of the LUX-Lung 3, 6 and 7 trials (afatinib), and from ARCHER 1050 (dacomitinib) also suggest that sequential treatment with second-generation TKIs followed by osimertinib may be feasible and effective. Among 37 patients who received osimertinib after afatinib in a pooled analysis of LUX-Lung 3, 6, and 7, the median time on osimertinib was 20.2 months and median overall survival (OS) was not reached after a median follow-up of $>4$ years; 3 -year OS was $>90 \%$ [13]. Likewise, in 22 patients who received a third-generation EGFR TKI following dacomitinib in ARCHER 1050, median OS was encouraging, at 36.7 months [3].

Real-world outcomes in 204 patients with T790M-positive NSCLC, who received sequential afatinib and osimertinib, were assessed in the recent retrospective, observational GioTag study [14]. Median time on treatment was 27.6 months overall, 30.3 months in patients with exon 19 deletion (Del19)-positive NSCLC, 36.4 months in patients with Del19-positive NSCLC with Eastern Cooperative Oncology Group (ECOG) performance status 0/1, and 46.7 months in Asian patients. The study enrolled a broad patient population, including patients with ECOG performance status $\geq 2$ (median time on treatment: 22.2 months) and patients with stable brain metastases (median: 19.4 months). These findings suggest that sequential afatinib and osimertinib can enable prolonged chemotherapy-free treatment in patients with T790M-acquired resistance, with a wide range of baseline characteristics.

Numerous factors must be acknowledged when considering the choice of EGFR TKI in any line of treatment. Given the high rate of CNS progression in patients with EGFRm + NSCLC, it is noteworthy that afatinib and osimertinib appear to penetrate the blood-brain barrier more effectively than first-generation TKIs (no data are available for dacomitinib). Indeed, both afatinib and osimertinib have shown activity against existing CNS lesions and in reducing the risk of CNS progression [5,15]. These data provide support for afatinib and osimertinib as first-line treatments of choice in patients with brain metastases, and suggest that sequential afatinib and osimertinib can be recommended for patients with and without CNS metastases [16].

Second-generation EGFR TKIs have a broader inhibitory profile than first-generation EGFR TKIs, which means that they are active against many uncommon and compound EGFR mutations, as well as common mutations (Del19/exon 21 substitution L858R) [9]. In addition to extending the range of patients for whom first-line afatinib is effective, its broader inhibitory profile may help to delay the expansion of heterogeneous resistant subclones during progression, resulting in more homogeneous T790M-positive clones compared with those that arise following resistance to first-generation EGFR TKIs. This is supported by preclinical observations [9] and could potentially contribute to prolonged responses to second-line osimertinib [17], as well as facilitating detection of the T790M mutation by tissue or liquid biopsy.

For patients with T790M-negative tumors, subsequent treatment options are more limited, although alternatives to standard platinum-based chemotherapy are now being identified. While immuno-oncology drugs have generally shown little benefit in patients with EGFRm + NSCLC, exploratory analysis of the Phase III IMPower150 study showed the combination of atezolizumab/bevacizumab/carboplatin/paclitaxel (ABCP) to have robust clinical activity after progression on EGFR TKIs [18]. Among 124 patients with EGFR mutations, OS and progression-free survival (PFS) were improved versus BCP, particularly in 91 patients with sensitizing EGFR mutations (OS, hazard ratio [HR]: 0.31; 95\% confidence interval [CI]: 0.11-0.83; PFS, HR: $0.41 ; 95 \%$ CI: $0.23-0.75)$. Specific data in patients with $\mathrm{T} 790 \mathrm{M}$-negative disease are needed to determine whether this treatment may be effective in this population. One regimen that has shown promise in patients with T790M-negative NSCLC is afatinib plus bevacizumab. In the Phase II ABC study in patients with EGFRm + NSCLC with acquired resistance to EGFR TKIs, treatment with afatinib/bevacizumab resulted in response rates of 14.3 and $22.2 \%$ in patients with T790Mpositive and T790M-negative tumors, respectively, with median PFS times of 6.3 and 7.1 months [19]. The afatinib/ bevacizumab combination could, therefore, be a salvage option for patients with T790M-negative NSCLC.

Of course, osimertinib is now established both as a first-line and a second-line option for the treatment of T790M-positive tumors, having demonstrated an impressive PFS benefit versus first-generation TKIs in the Phase III FLAURA trial [2]. Nevertheless, it is important to consider that mechanisms of resistance to osimertinib are 
more heterogeneous than for first- and second-generation EGFR TKIs. Next-generation sequencing analysis of FLAURA samples indicated that the most common mechanism of resistance to first-line osimertinib $(\mathrm{n}=91)$ was MET amplification (15\%), followed by C797S in 7\%, but no putative resistance mechanism was identified in $>60 \%$ of tumors [20]. Thus, currently, no standard targeted treatment is available following osimertinib failure, and most patients will proceed to chemotherapy. This has led physicians to question whether long-term benefit could be better achieved by reserving osimertinib for second-line use, to prolong the chemotherapy-free period. However, it must be acknowledged that not all patients will develop T790M-positive tumors, and a proportion (i.e., those with T790M-independent resistance mechanisms) would not be eligible to receive and benefit from osimertinib if it was not given up front [5]. Thus, there is a risk in retaining osimertinib for second-line use. If a sequencing approach is adopted, the patient must be monitored closely for disease progression, and all possible testing methods for T790M must be applied.

In summary, further research is needed to define the optimal treatment sequence to maximize long-term survival, while maintaining quality of life. This will be facilitated by further development of highly sensitive and specific, noninvasive diagnostic techniques to optimize EGFR mutation testing.

\section{Author contributions}

MJ Hochmair was responsible for the conception and design of the work, participated in drafting the work or revising it critically for important intellectual content, approved the submitted manuscript and agrees to be accountable for all aspects of the work.

Financial \& competing interests disclosure

MJ Hochmair has received honoraria from AstraZeneca, Bristol-Myers Squibb, Boehringer Ingelheim, Merck Sharp \& Dohme, Pfizer, and Roche, and has had consulting or advisory roles with Boehringer Ingelheim, Merck Sharp \& Dohme, Novartis and Roche. The authors have no other relevant affiliations or financial involvement with any organization or entity with a financial interest in or financial conflict with the subject matter or materials discussed in the manuscript apart from those disclosed.

Medical writing support was funded by Boehringer Ingelheim Inc. and provided by Fiona Scott, PhD, CMPP of GeoMed, an Ashfield company, part of UDG Healthcare plc.

\section{Open access}

This work is licensed under the Attribution-NonCommercial-NoDerivatives 4.0 Unported License. To view a copy of this license, visit http://creativecommons.org/licenses/by-nc-nd/4.0/

\section{References}

1. Park K, Tan EH, O'Byrne K et al. Afatinib versus gefitinib as first-line treatment of patients with EGFR mutation-positive non-small-cell lung cancer (LUX-Lung 7): a Phase 2B, open-label, randomised controlled trial. Lancet Oncol. 17, 577-589 (2016).

2. Soria JC, Ohe Y, Vansteenkiste J et al. Osimertinib in untreated EGFR-mutated advanced non-small-cell lung cancer. $N$. Engl. J. Med. 378, 113-125 (2018).

3. Mok TS, Cheng Y, Zhou X et al. Improvement in overall survival in a randomized study that compared dacomitinib with gefitinib in patients with advanced non-small-cell lung cancer and EGFR-activating mutations. J. Clin. Oncol. 36, 2244-2250 (2018).

4. Yang JC, Ahn MJ, Kim DW et al. Osimertinib in pretreated T790M-positive advanced non-small-cell lung cancer: AURA Study Phase II extension component. J. Clin. Oncol. 35, 1288-1296 (2017).

5. Girard N. Optimizing outcomes in EGFR mutation-positive NSCLC: which tyrosine kinase inhibitor and when? Future Oncol. 14, 1117-1132 (2018).

6. Mok TS, Wu YL, Ahn MJ et al. Osimertinib or platinum-pemetrexed in EGFR T790M-positive lung cancer. N. Engl. J. Med. 376, 629-640 (2017).

7. Buder A, Hochmair MJ, Schwab S et al. Cell-free plasma DNA-guided treatment with osimertinib in patients with advanced EGFR-mutated NSCLC. J. Thorac. Oncol. 13, 821-830 (2018).

8. Hochmair MJ, Buder A, Schwab S et al. Liquid-biopsy-based identification of EGFR T790M mutation-mediated resistance to afatinib treatment in patients with advanced EGFR mutation-positive NSCLC, and subsequent response to osimertinib. Target. Oncol. 14, 75-83 (2019).

9. Kohsaka S, Petronczki M, Solca F, Maemondo M. Tumor clonality and resistance mechanisms in EGFR mutation-positive non-small-cell lung cancer: implications for therapeutic sequencing. Future Oncol. 15, 637-652 (2019).

10. Rolfo C, Mack PC, Scagliotti GV et al. Liquid biopsy for advanced non-small-cell lung cancer (NSCLC): a statement paper from the IASLC. J. Thorac. Oncol. 13, 1248-1268 (2018).

11. Diaz LA Jr, Bardelli A. Liquid biopsies: genotyping circulating tumor DNA. J. Clin. Oncol. 32, 579-586 (2014). 
12. Buder A, Setinek U, Hochmair MJ et al. EGFR mutations in cell-free plasma DNA from patients with advanced lung adenocarcinomas: improved detection by Droplet Digital PCR. Target. Oncol. 14, 197-203 (2019).

13. Sequist L, Wu Y-L, Schuler M et al. Subsequent therapies post-afatinib among patients (pts) with EGFR mutation-positive (EGFRm+) NSCLC in LUX-Lung (LL) 3, 6 and 7. Ann. Oncol. 28, 482-483 (2017).

14. Hochmair M, Cufer T, Morabito A et al. Sequential treatment with afatinib and osimertinib in real-world patients with EGFR mutation-positive advanced NSCLC: the GioTag study. Lung Cancer 127, S37 (2019).

15. Reungwetwattana $\mathrm{T}$, Nakagawa $\mathrm{K}$, Cho BC et al. CNS response to osimertinib versus standard epidermal growth factor receptor tyrosine kinase inhibitors in patients with untreated EGFR-mutated advanced non-small-cell lung cancer. J. Clin. Oncol. 36, 3290-3297 (2018).

16. Hochmair M. Medical treatment options for patients with epidermal growth factor receptor mutation-positive non-small cell lung cancer suffering from brain metastases and/or leptomeningeal disease. Target. Oncol. 13, 269-285 (2018).

17. Tamiya M, Tamiya A, Suzuki $\mathrm{H}$ et al. Which of afatinib and gefitinib/erlotinib is the better EGFR-TKI to be followed by osimertinib? Ann. Oncol. 29, viii527-viii528 (2018).

18. Mok TSK, Socinski MA, Reck M et al. IMpower150: an exploratory analysis of efficacy outcomes in patients with EGFR mutations. Ann. Oncol. 29, ix173-ix178 (2018).

19. Hata A, Katakami N, Kaji R et al. Afatinib plus bevacizumab combination after acquired resistance to EGFR tyrosine kinase inhibitors in EGFR-mutant non-small cell lung cancer: multicenter, single-arm, Phase 2 trial (ABC Study). Cancer 124, 3830-3838 (2018).

20. Ramalingam SS, Rukazenkov Y, Todd A et al. Mechanisms of acquired resistance to first-line osimertinib: preliminary data from the Phase III FLAURA study. Ann. Oncol. 29, viii740 (2018). 